\title{
Combined oral anticoagulant and antiplatelet treatment: need for an evidence-based approach
}

\author{
Marco Moia
}

Received: 12 May 2010/Accepted: 25 May 2010/Published online: 24 June 2010

(C) SIMI 2010

Oral anticoagulants and antiplatelets are used in the longterm treatment and prevention of thromboembolic diseases. For more than 50 years, vitamin $\mathrm{K}$ antagonists (VKA), i.e. warfarin, acenocoumarol, and phenprocoumon, have been the only available oral anticoagulants. New oral anticoagulant drugs will soon be available: those at a more advanced stage of development are a direct thrombin inhibitor (dabigatran etexilate) and two direct factor $\mathrm{Xa}$ inhibitors (rivaroxaban and apixaban). Antiplatelet drugs interfere with some steps in the activation process, including adhesion, release, and/or aggregation. Aspirin and thienopyridines (ticlopidine and clopidogrel) are the currently used drugs, but new molecules are coming. VKA are used in the primary and secondary prevention of venous thromboembolism, in the prophylaxis of cardioembolic events in patients with mechanical or biological prosthetic heart valves, in patients with atrial fibrillation, and in some other ischemic myocardial diseases; antiplatelets are used mainly to prevent and/or to treat myocardial infarction, stroke, and peripheral arterial diseases.

Combined anticoagulant-antiplatelet treatment has been attempted in order to increase the efficacy of the antithrombotic treatment in some clinical conditions. Moreover, one patient already on anticoagulant treatment may develop also the indication for antiplatelet treatment, or vice versa. For both anticoagulants and antiplatelets, the efficacy in treating and preventing thromboembolic diseases cannot be dissociated from an increased risk of bleeding; therefore, the combination of these drugs can be

\section{Moia $(\square)$}

Angelo Bianchi Bonomi Hemophilia and Thrombosis Center, Fondazione IRCCS Ca' Granda, Ospedale Maggiore Policlinico, Via Pace 9, 20122 Milan, Italy

e-mail: moia@policlinico.mi.it not only more effective, but also more dangerous, and should be considered with caution. Overall, the evidence of benefit of combined treatment is limited, and the increased risk of bleeding is proven. However, combined anticoagulant and antiplatelet treatment is frequently prescribed in clinical practice: an aggressive therapeutic approach seems currently to be more attractive and popular than an evidence-based one. Sometimes it may be difficult to assess the benefit-risk profile of combined anticoagulant-antiplatelet treatment versus the use of a single antithrombotic agent. For the above-mentioned reasons, the excellent review by Vedovati et al. [1], published in this issue of Internal and Emergency Medicine, can be very helpful in clinical practice. The authors made an extensive and critical perusal of the available studies and come to well documented, sound conclusions. To summarize, the indications for combined anticoagulant-antiplatelet treatment are limited. VKA and aspirin is the only combined anticoagulant-antiplatelet treatment which was extensively investigated. There is evidence that this association increases the risk of bleeding: it may have a favorable benefit-risk profile in patients with mechanical prosthetic heart valves or in patients with coronary artery disease. However, also among patients with mechanical valves or coronary disease, a selection should be made in order to assess the individual risk-benefit profile; a special attention should always be paid to the risk of bleeding. When combined treatment is prescribed, low doses of aspirin should be used, and tight INR monitoring is required. In case of a poor anticoagulation control, corrective actions should promptly be started, i.e. patient training, more frequent monitoring, or even reconsidering the therapeutic strategy. Indications and contra-indication should be periodically reconsidered in each patient on combined treatment. Vedovati et al. [1] point out that no clear advantage 
of the combined treatment has been demonstrated in patients with atrial fibrillation, which is by far the most frequent indication for VKA. Phase III trials investigating new anticoagulants such as idraparinux [2] and ximelagatran [3, 4] also provide evidence about the increased risk of bleeding due to combined VKA-aspirin treatment in patients with atrial fibrillation. In the warfarin arm of the Amadeus trial [2], patients who received aspirin in combination with warfarin had almost double the rate of clinically relevant bleeding than patients who received warfarin alone (17.7 vs. 9.3\%). An increased overall bleeding was also reported in patients receiving aspirin in the warfarin arms of SPORTIF III trial [3] (52.1 vs. 26.3\%) and SPORTIF V trial [4] (69 vs. 44\%). We recently surmised [5] that the unusually high incidence of major bleeding in the warfarin arm of the RELY trial with dabigatran [6] may be in part attributable to the high percentage (about $20 \%$ ) of patients concomitantly treated with aspirin. What is evident from all the above-mentioned studies is that the proportion of patient with atrial fibrillation who receive combined warfarin and aspirin treatment has been increasing in the recent years: from $10 \%$ in SPORTIF III trial [3], published in 2003, to $15 \%$ in SPORTIF V trial [4] (2005), to 20 and $21 \%$, respectively, in Amadeus trial [2] and RELY trial [6] (2008 and 2009, respectively). Since no clear advantage of the combined treatment has been shown in patients with atrial fibrillation, this trend (which mirrors my personal impression in daily clinical practice) is not evidence based.

Antiplatelet drugs different from aspirin, namely clopidogrel, could cause even higher risk of severe bleeding when combined with VKA. A recent retrospective analysis of nationwide registry in Denmark [7] found that the yearly incidence of bleeding, defined as an admission to hospital with a diagnosis of non-fatal or fatal bleeding, was $4.3 \%$ for VKA, $5.1 \%$ for aspirin plus VKA, $12.3 \%$ for clopidogrel plus VKA, and $12.0 \%$ for triple therapy. Increased risk of bleeding was associated with previous bleeding, older age ( $>70$ years), diabetes, and heart failure. Thus, this study may also provide some useful hints for selecting patients in whom combined oral anticoagulants and antiplatelets should not be used.

Preliminary data suggest that combined treatment with dabigatran and aspirin would not significantly increase the risk of major bleeding, if compared with dabigatran alone [8]. Therefore, it is conceivable that the combination of new anticoagulants with (new) antiplatelets may offer a better safety/risk profile than VKA combined with aspirin or clopidogrel. However, this hypothesis needs to be carefully tested with specific studies. Taking into account that many new anticoagulants and antiplatelets will soon be available, combined treatment will become a hot and complex topic.

In conclusion, the review by Vedovati et al. [1] will be certainly useful for the physician in order to reconsider his trend in prescribing combined anticoagulant-antiplatelet treatment. I hope that caution will be used in daily clinical practice: we should be aware that a serious bleeding event requires the interruption of any anticoagulant and antiplatelet drug (sometimes for long periods), exposing the patient to the subsequent high risk of thromboembolic/ ischemic events. A "primum non nocere" approach is advisable whenever the benefit of the combined anticoagulant-antiplatelet treatment is uncertain.

Conflict of interest None.

\section{References}

1. Vedovati MC, Becattini C, Agnelli G (2010) Combined oral anticoagulants and antiplatelets: benefits and risks. Intern Emerg Med. doi:10.1007/s11739-010-0349-x

2. Amadeus Investigators, Bousser MG, Bouthier J et al (2008) Comparison of idraparinux with vitamin $\mathrm{K}$ antagonists for prevention of thromboembolism in patients with atrial fibrillation: a randomised, open-label, non-inferiority trial. Lancet 371:315321

3. Olsson SB (2003) Stroke prevention with the oral direct thrombin inhibitor ximelagatran compared with warfarin in patients with non-valvular atrial fibrillation (SPORTIF III): randomised controlled trial. Lancet 362:1691-1698

4. Albers GW, Diener HC, Frison L, Grind M, Nevinson M, Partridge $\mathrm{S}$, Halperin JL, Horrow J, Olsson SB, Petersen P, Vahanian A, SPORTIF Executive Steering Committee for the SPORTIF V Investigators (2005) Ximelagatran vs warfarin for stroke prevention in patients with nonvalvular atrial fibrillation: a randomized trial. JAMA 293:690-698

5. Moia M, Mannucci PM (2009) Dabigatran versus warfarin in patients with atrial fibrillation. N Engl J Med 361:2672

6. Connolly SJ, Ezekowitz MD, Yusuf S et al (2009) Dabigatran versus warfarin in patients with atrial fibrillation. N Engl J Med 61:1139-1151

7. Sorensen R, Hansen ML, Abildstrom SZ et al (2009) Risk of bleeding in patients with acute myocardial infarction treated with different combinations of aspirin, clopidogrel, and vitamin $\mathrm{K}$ antagonist in Denmark: a retrospective analysis of nationwide registry data. Lancet 374:1967-1974

8. Ezekowitz MD, Reilly PA, Nehmiz G, Simmers TA, Nagarakanti R, Parcham-Azad K, Pedersen KE, Lionetti DA, Stangier J, Wallentin L (2007) Dabigatran with or without concomitant aspirin compared with warfarin alone in patients with nonvalvular atrial fibrillation (PETRO study). Am J Cardiol 100:1419-1426 